\title{
Influence of pollution and extraneous inclusions on the scattering of THz radiation by fabric
}

\author{
$\underline{\text { A. A. Angeluts }}{ }^{1}$, V. N. Aksenov ${ }^{1}$, A. V. Balakin ${ }^{1}$, I. A. Ozheredov ${ }^{1}$, A. P. Shkurinov ${ }^{1}$ \\ ${ }^{1}$ Faculty of Physics M.V.Lomonosov Moscow State University, Moscow, Russia, angeluts@ physics.msu.ru
}

One of the challenges of modern civilization now is the various manifestations of terrorist activity. It is therefore necessary to create different methods of remote diagnostics of dangerous items and substances. One of directions of development of diagnostic methods introduced in recent decades is methods based on the use of Terahertz $(\mathrm{THz})$ radiation.

$\mathrm{THz}$ radiation is defined as electromagnetic radiation in the range from $300 \mathrm{GHz}$ to $10 \mathrm{THz}$ (or $30 \mu \mathrm{m}$ to $1 \mathrm{~mm}$ ), is of great interest to create diagnostic, measuring and inspection systems. This interest determines firstly by the fact that in recent years, started an intensive development of components for the generation and detection of $\mathrm{THz}$ radiation, and secondly, the specific features of the radiation. Among the important properties of this radiation should be noted his non-invasive and non-ionizing nature that allows the use of $\mathrm{THz}$ radiation in tasks related to human Diagnostics. In addition, this radiation weakly attenuated by most materials, so such objects, such as cloth, dry wood, paper, plastics are transparent to $\mathrm{THz}$ range.

Most often, the diagnostic is to irradiate the $\mathrm{THz}$ object of interest by probe beam and then analyze the transmitted, reflected or scattered radiation.

In the present work describes a study, based on analysis of the scattered radiation.

\section{Experimental setup and samples}

As the object of the study, tissue samples were selected with TNT traces on their surfaces or that contain a thin metal wire. Such objects simulate a clothes whose surface is contaminated with a hazardous substance or contains elements of electronic devices controlling the explosive device (current leads, antenna elements and so on).

Studies performed with the setup, the simplified diagram is shown in Fig.1. Our experimental setup detailed description can be found in [1]. Radiation with a frequency of $3 \mathrm{THz}$ focused on the surface of the object along normal to its surface. The scattered radiation was collected by a system of parabolic mirrors and detected. Recorded radiation scattered at an angle of approximately 60 degrees from the normal, or 120 degrees from the direction of incidence of the probe beam. This angle allows the detector to avoid specular component of the radiation.

Samples were flaps of cotton fabric. "Contamination" of the sample was carried out by applying TNT solution in acetone to the tissue. Then the sample was dried and weighed.

The sample $C t 14$ had a surface contamination density of about $3 \mathrm{mg} / \mathrm{cm}^{2}$.
The sample $C t 50$ had a surface contamination density of $10 \mathrm{mg} / \mathrm{cm}^{2}$.

THz beam
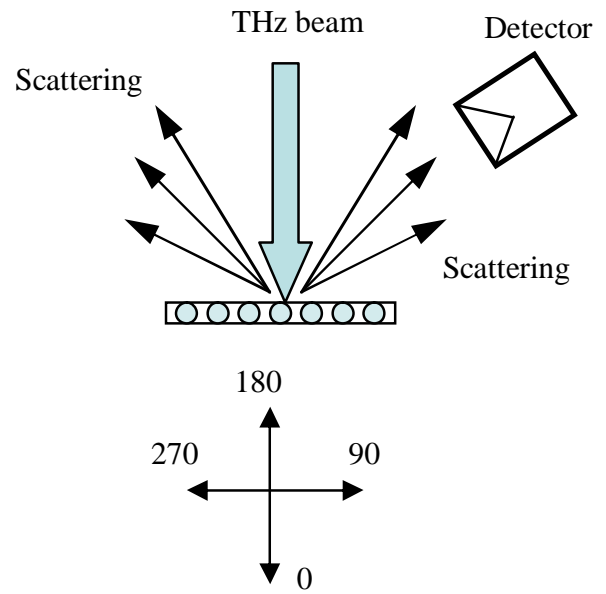

Fig. 1. Scattering experiment schematic

Since no uniform contamination was achieved in the preparation of samples, the above figures only approximately characterize the degree of contamination in the analyzed sample area.

Cm78 sample consisted of a piece of cloth of the same, in which a copper wire of $78 \mathrm{mkm}$ was introduced.

In addition to the samples described above, samples were studied that were copper wires 240 and 78 $\mathrm{mkm}$ in diameter. With their help it was conducted an adjustment of the measuring circuit.

\section{Experimental results}

Table shows some of the results of the measurements represent the values of ambient signal from different samples. For ease of comparison values of the scattering signal, it was normalized to the value of the scattering signal of pure textiles.

Table . Relative magnitude of scattering for different samples

\begin{tabular}{|l|l|l|l|l|l|l|}
\hline Sample & $\begin{array}{l}\text { Copper } \\
\text { wire } \\
\phi 78 \\
\mathrm{mkm}\end{array}$ & $\begin{array}{l}\text { Copper } \\
\text { wire } \\
\phi 240 \\
\mathrm{mkm}\end{array}$ & $\mathrm{Ct14}$ & $\mathrm{Ct50}$ & Textile & $\mathrm{Cm} 78$ \\
\hline $\begin{array}{l}\text { Scat- } \\
\text { tering } \\
\text { signal } \\
\text { value }\end{array}$ & 5.4 & 18.3 & 0.8 & $\begin{array}{l}0.17- \\
0.58\end{array}$ & 1 & 1.75 \\
\hline
\end{tabular}

The signal value for sample $C t 50$ shows a spread due to strong heterogeneity of pollution, as when drying solution came the transfer of a substance to the periphery of the sample. So most likely score pollution concentration overstated 
As can be seen from the table, tissue contamination leads to suppression of scattering signal in our choice of angle observation from $20 \%$ up to 5 times. The magnitude of the suppression is determined by the concentration of the substance in the area under study

The presence of thin conductor reliably detected in tissue. The copper wire with a diameter of $78 \mathrm{mkm}$ sewn into the fabric leads to an increase in the scattered signal by a factor of 1.8 .

The scattered signal from a single conductor exceeds the signal from the surface of the cotton fabric from 5 to 20 times, depending on the size of the conductor

The figure 2 shows the result of yet another experiment. Here, the sample $\mathrm{Cm} 78$ moved along the surface plane, simulating the scanning process of the sample. The width of the curve in Fig. 2 is one and a half times smaller than the $\mathrm{THz}$ size of the spot on the surface. The "pedestal" of the chart is determined by the scattering from the tissue itself. A slight decrease in the magnitude of the signal at the positions 5 and 6 $\mathrm{mm}$ is caused by the destructive interference of the radiation scattered by the tissue and the conductor.

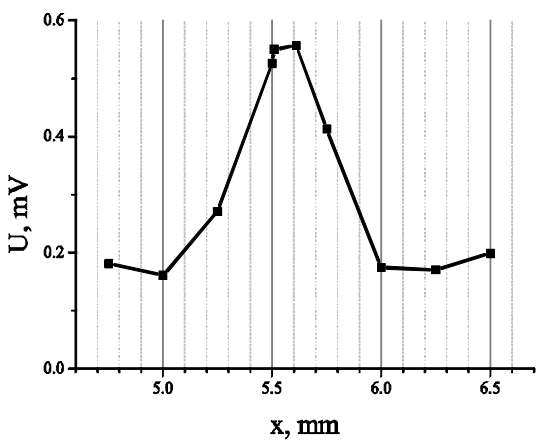

Fig. 2. The result of the movement of $\mathrm{Cm} 78$ sample across the probing beam. Detection of hidden wire.

\section{Scattering numerical estimation}

A numerical estimate of the scattering was carried out on the simplest model object-an infinite cylinder covered by a layer of an absorbing dielectric [2].. This model most closely describes the textile, which is a set of threads.

In our model for base cylinder, we used parameters of PET (also known as Lavsan) and cotton (see Fig. 3 and 4). The diameter of the threads equals to 100 microns. As a coating, TNT layers of different thicknesses were considered: $0.1,1$ and $10 \mu \mathrm{m}$.

Figures 3 and 4 show fragments of the scattering indicatrix for model objects. The angle of 180 degrees corresponds to the direction of backscattering of the incident radiation. On the graphs given, the value of the scattering signal is normalized to the value for the uncoated strand.

Given charts show that the nature of the scattering can vary significantly with increasing thickness of a polluted layer. Strong pollution (layer $10 \mathrm{mkm}$ ) leads to the Suppression of scattering that qualitatively corresponds to experiment. Under certain conditions
$(1 \mathrm{mkm})$ scattering may even increase. A small contamination (layer $100 \mathrm{~nm}$ ) changes the magnitude of the signal is less than $10 \%$.

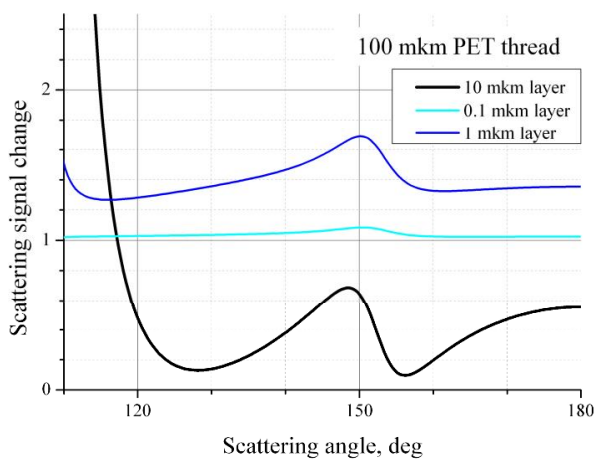

Fig. 3. The change of the scattering indicatrix with the variation in the thickness of the polluting layer on PET thread

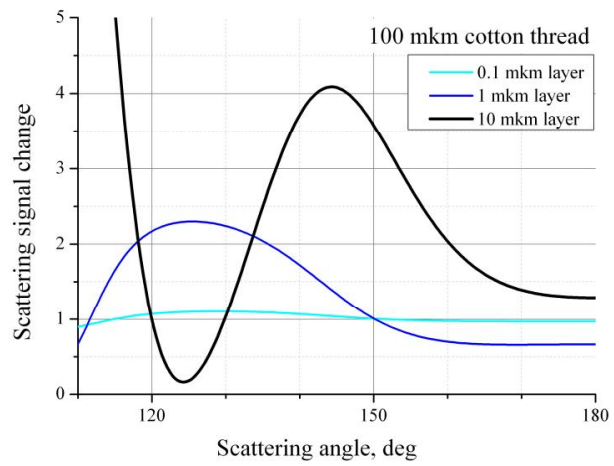

Fig. 4. The change of the scattering indicatrix with the variation in the thickness of the polluting layer on cotton thread

\section{Conclusion}

The possibility of detecting current-carrying elements or traces of explosives on the surface of the object by analyzing scattered $\mathrm{THz}$ radiation is investigated.

Experimental results and preliminary calculations have shown that the backscattering patterns for textile fibers differ significantly, depending on their material and the presence of contamination on them. This opens up the possibility of developing terahertzimaging systems in the field of security.

\section{Acknowledgements}

This work was supported by the Russian Foundation for Basic Research (project No. 16-29-09593)

\section{References}

1. Aksenov, V. N., Angeluts, A. A., Balakin., A. V. et. al. Application of a terahertz multi-frequency radiation source based on quantum-cascade lasers for identification of substances basing on the amplitude-spectral analysis of the scattered field // Radiophysics and Quantum Electronics. 2018. V. 60, No. 11. P. 877-888

2. Kotlar, V. V., Lichmanov, M. A. Analysis of the diffraction of an electromagnetic wave on an infinite circular cylinder with several homogeneous layers. Computer Optics (in rus.). 2002. V. 24. P. 26-23 